\title{
Fabrication of photonic crystals in rare-earth doped chalcogenide glass films for enhanced upconversion
}

\author{
M. E. Pollard ${ }^{a}$, K. J. Knight ${ }^{c}$, G. J. Parker ${ }^{a}$, D. W. Hewak ${ }^{b}$, and M. D. B. Charlton ${ }^{a}$ \\ ${ }^{a}$ Nano Research Group, School of Electronics and Computer Science, University of \\ Southampton, Highfield, Southampton, UK, SO17 1BJ; \\ ${ }^{b}$ Optoelectronics Research Centre, University of Southampton, \\ Highfield, Southampton, UK, SO17 1BJ; \\ ${ }^{c}$ School of Chemistry and Physics, University of Adelaide, SA 5005, Australia
}

\begin{abstract}
Gallium lanthanum oxysulfide (GLSO) is a promising host material for observing strong upconversion emission from trivalent rare-earth ions such as erbium $\left(\mathrm{Er}^{3+}\right)$. Its attractive properties include high rare-earth solubility due to the lanthanum content of the glass former, a high refractive index $(n=2.2$ at $550 \mathrm{~nm})$ for high radiative efficiency, and a low maximum phonon energy of approximately $425 \mathrm{~cm}^{-1}$. Photonic crystals meanwhile can provide controlled light extraction, and may be capable of suppressing unwanted IR emission from lower lying metastable states. Here, we describe the fabrication of photonic crystals in annealed films of Er ${ }^{3+}$-doped GLSO deposited by RF sputtering. The most intense visible upconversion emission is observed in films annealed at $550^{\circ} \mathrm{C}$, close to the bulk glass transition temperature. Hexagonal lattice photonic crystals are subsequently milled into the films using a focused ion beam (FIB). The milling parameters are optimized to produce the most vertical sidewall profile.
\end{abstract}

Keywords: Photonic crystal, upconversion, rare earth, erbium, chalcogenide, gallium lanthanum sulfide, GLS

\section{INTRODUCTION}

Upconversion lasers based on the higher lying states of rare-earth (RE) ions are a potential solution for compact devices emitting at visible wavelengths, not only to fill the green gap left by semiconductor laser diodes but to act as complete RGB sources. Two promising low phonon energy hosts for RE ions are gallium lanthanum sulfide (GLS) and its more thermally-stable variant gallium lanthanum oxysulfide (GLSO). Strong visible upconversion has been observed in $\mathrm{Er}^{3+}: \mathrm{GLS}^{1}$ and $\mathrm{Er}^{3+}: \mathrm{GLSO}^{2}$ on the $\left\{{ }^{2} H_{11 / 2},{ }^{4} S_{3 / 2}\right\} \rightarrow{ }^{4} I_{15 / 2}(550 \mathrm{~nm}, 525 \mathrm{~nm})$ and ${ }^{4} F_{9 / 2} \rightarrow$ ${ }^{4} I_{15 / 2}(670 \mathrm{~nm})$ transitions. As with $\mathrm{Er}^{3+}$-doped fiber amplifiers and lasers operating around 1550nm, pumping efficiency at $980 \mathrm{~nm}$ can be dramatically improved by co-doping with $\mathrm{Yb}^{3+}$ ions. $^{2}$ However, the pumping efficiency for upconversion lasers still remains much lower than for conventional lasers.

Here, we report the fabrication of photonic crystals $(\mathrm{PhCs})$ in sputtered films of $\mathrm{Er}^{3+}$ :GLSO for the express purpose of demonstrating enhanced visible upconversion mediated by suppressed IR emission from the intermediate states. In Sec. 2 we introduce the favorable properties of GLSO for upconversion processes, practical challenges, and the benefits of PhCs for upconversion lasers. Section 3 describes the deposition, annealing, and patterning of the Er:GLSO thin films, including optimization of the FIB milling process. Fluorescence measurements on the unpatterned films are presented in Sec. 4, with concluding remarks in Sec. 5.

\section{RELEVANT PROPERTIES OF THE HOST AND PHOTONIC CRYSTALS}

\subsection{Gallium Lanthanum Sulfide Glasses}

Gallium lanthanum oxysulfide (GLSO) is a chalcogenide glass with a transmission window stretching from $10 \mu \mathrm{m}$ to approximately $0.5 \mathrm{\mu m}$. The significant visible transparency, which is unusual for chalcogenides, can be extended by the addition of halide glass modifiers. ${ }^{1,3}$ The maximum phonon energy in GLS is approximately

Send correspondence to M. E. Pollard (E-mail: mep06r@ecs.soton.ac.uk, Telephone: +44 (0)23 80593127 ) 
$425 \mathrm{~cm}^{-1}$, permitting efficient upconversion ${ }^{1}$ with dopant species including $\mathrm{Er}^{3+}, \mathrm{Nd}^{3+}, \mathrm{Pr}^{3+}$, and $\mathrm{Ho}^{3+}$. In addition, the lanthanum content allows high RE doping without concentration quenching, and the amorphous structure broadens absorption linewidths, thereby increasing the chances of monochromatic pumping schemes. Furthermore, the relatively high refractive index of GLSO $(n=2.2$ at 550nm) ensures high radiative efficiency.

Practical drawbacks of GLS-based hosts include the still limited visible transparency and potentially damaging photosensitivity. Their low thermal conductivity and strong thermal lensing also necessitate the use of geometries capable of efficiently dissipating heat, such as optical fibers or planar waveguides. However, patterning GLSbased glasses is complicated by their resistance to standard dry etch processes. Even lift-off is difficult since the only physical vapor deposition (PVD) techniques known to be compatible with GLS are sputtering ${ }^{4}$ and pulsed laser deposition (PLD). ${ }^{5,6}$ Both methods tend to be conformal, often making patterned features inseparable from the photoresist. ${ }^{7}$ Some authors attempt to circumvent this issue using either long-throw or collimated sputtering, at the expense of a reduced deposition rate. ${ }^{8}$ Others exploit the inherent photosensitivity of GLS to write features directly with UV lasers. ${ }^{9}$ In similar glass sulfides, there have been demonstrations of more exotic techniques such as hot embossing, ${ }^{10-12}$ or photodissolution of silver, ${ }^{13,14}$ which also has the potential to increase radiative efficiency through the local field enhancement of metal nanoparticles. ${ }^{15}$

\subsection{Enhanced Fluorescence in Photonic Crystals}

Photonic crystals (PhCs) can modify the local density of states of the host material and control spontaneous emission. ${ }^{16}$ Although there are many groups citing control of Stokes luminescence with PhCs*, there are very few who have studied anti-Stokes (upconversion) emission. To date, most of these have focused on band-edge enhancement and mid-gap suppression of the visible emission by the presence of a photonic band gap (PBG) in the same spectral range. ${ }^{18-20}$

Russell $^{21}$ and Withnall et al. ${ }^{22}$ both suggested an alternative approach to highly efficient upconversion pumping by using the PBG to suppress IR emission from the lower lying metastable states. Low phonon energy hosts are already used to reduce non-radiative transitions, therefore reducing unwanted radiative transitions appears to be the next logical step. The proposal is supported by recent experimental work modifying the lifetime of the $\left\{{ }^{2} H_{11 / 2},{ }^{4} S_{3 / 2}\right\}$ states in Er:Yb:NaYF 4 nanoparticles. ${ }^{18}$ Applying the same principle to the strong ${ }^{4} I_{13 / 2} \rightarrow{ }^{4} I_{15 / 2}(1.538 \mu \mathrm{m})$ transition in $\mathrm{Er}^{3+}$ could increase the probability of excitation to $\left\{{ }^{2} H_{11 / 2},{ }^{4} S_{3 / 2}\right\}$ $(525 \mathrm{~nm}, 550 \mathrm{~nm})$ by excited state absorption (ESA) resonant at a pump wavelength near 800nm. One can even imagine employing a biperiodic $\mathrm{PhC}$, or PhCs with higher order PBGs, to combine suppression of IR emission with band-edge enhancement of visible emission.

PhCs can also support high-Q defect and band-edge mode resonances that are, in theory, comparable to microring or microsphere resonators. ${ }^{23,24}$ The huge benefit of high-Q resonators for upconversion lasers is exemplified by Lu et al. ${ }^{24}$ who demonstrated low-threshold lasing on the ${ }^{4} S_{3 / 2} \rightarrow{ }^{4} I_{15 / 2}$ transition in a 0.1 $0.5 \mathrm{~mol} \% \mathrm{Er}_{\mathrm{SiO}} \mathrm{SiO}_{2}$ toroidal microcavity $\left(\mathrm{Q}>10^{7}\right)$, despite silica's high maximum phonon energy of $\sim 1100 \mathrm{~cm}^{-1}$.

\section{FABRICATION OF PHOTONIC CRYSTALS IN GLSO}

\subsection{Glass Melts for Sputtering Targets}

GLS, GLSO, and 0.2 wt\% Er:GLSO sputtering targets were produced in-house using commercially available powdered precursors. The relatively low concentration of the doped sample was chosen to prevent concentration quenching inhibiting future measurements of the upper state lifetimes, and to limit ion-ion interactions that can enhance or suppress upconversion through cross relaxation. The molar compositions of the pure GLS and GLSO targets were $65 \mathrm{Ga}_{2} \mathrm{~S}_{3}: 32 \mathrm{La}_{2} \mathrm{~S}_{3}: 3 \mathrm{La}_{2} \mathrm{O}_{3}$, and $76.51 \mathrm{Ga}_{2} \mathrm{~S}_{3}: 23.49 \mathrm{La}_{2} \mathrm{O}_{3}$, respectively. The small percentage of $\mathrm{La}_{2} \mathrm{O}_{3}$ in the GLS target ensured successful vitrification. For the Er:GLSO target, $0.13 \mathrm{~mol}_{\%}$ of $\mathrm{La}_{2} \mathrm{O}_{3}$ was replaced with $\mathrm{Er}_{2} \mathrm{O}_{3}$, to give an $\mathrm{Er}^{3+}$ ion density of $\sim 2.519 \times 10^{19} \mathrm{~cm}^{-3}$. Appropriate amounts of each precursor were gently mixed in PTFE jars in a dry nitrogen purged glovebox. The powders were then transferred to vitreous carbon crucibles and melted at $1100^{\circ} \mathrm{C}$ for 22 hours in a horizontal tube furnace with a flowing Ar atmosphere $\left(500 \mathrm{ml} \mathrm{min}^{-1}\right)$. Once quenched and cooled, the glass was cleaned with solvents, crushed with a mortar and pestle, and transferred to a 2 inch diameter carbon cylinder. The crushed glass was re-melted in a vertical tube

${ }^{*}$ See, for example, Noda et al. ${ }^{17}$ and references therein. 


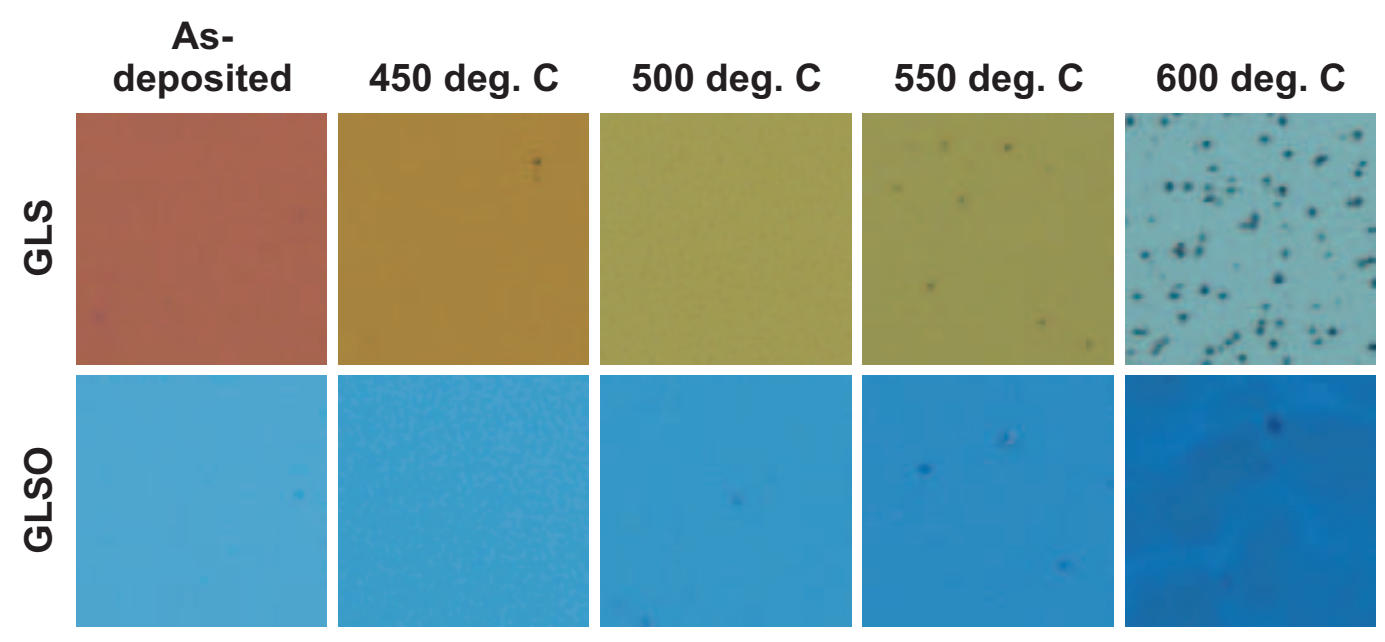

Figure 1. Microscope images (20x) of thin GLS and GLSO films deposited on thermally-oxidized silicon in the as-deposited state, and annealed for 2 hours in $\mathrm{Ar}$ at temperatures between $450^{\circ} \mathrm{C}$ and $600^{\circ} \mathrm{C}$.

furnace at a slightly higher temperature of $1170^{\circ} \mathrm{C}$ for 4 hours in flowing $\mathrm{Ar}\left(5000 \mathrm{ml} \mathrm{min}^{-1}\right)$. The Er:GLSO target was made by adding the necessary amount of $\mathrm{Er}_{2} \mathrm{O}_{3}$ to the crushed glass prior to the second melt. After quenching, the resulting 2 inch glass ingots were slowly annealed for 24 hours at $510^{\circ} \mathrm{C}$ in $\mathrm{Ar}\left(1500 \mathrm{ml} \mathrm{min}^{-1}\right)$, using up and down ramp rates of $1^{\circ} \mathrm{C} \mathrm{min}^{-1}$. Sputtering targets approximately $3 \mathrm{~mm}$ thick were cut from the glass ingots and mounted on copper backing plates with silver-loaded epoxy.

\subsection{Sputtering and Annealing of Thin Films}

To date, three methods have been used to deposit thin films of GLS-based glasses: pulsed laser deposition, ${ }^{5,6,25}$ hot dip spin coating, ${ }^{25,26}$ and sputtering. ${ }^{4}$ All three methods are well-suited to deposition of multicomponent glasses. In this work, sputtering was chosen for its flexibility, high thickness and compositional uniformity, accurate stoichiometry, and substrate independence.

Thin films of GLS (162nm), GLSO (100nm), and Er:GLSO (150nm) were deposited on 2 inch silicon wafers with a $2 \mu \mathrm{m}$ thermal oxide by RF sputtering. The film thickness for the Er:GLSO was chosen to ensure singlemoded operation and minimize the impact of higher order guided modes on the width or existence of a guided mode PBG. Deposition was performed without substrate heating in a sputter-down configuration with a base pressure of $3 \mathrm{mT}$, Ar flow rate of $15 \mathrm{sccm}$, and an energy density of approximately $3 \mathrm{~W} \mathrm{~cm}$. The rate for all targets was approximately $1 \mathrm{~nm} \mathrm{~min}{ }^{-1}$. After deposition, five $10 \times 10 \mathrm{~mm}$ pieces were cleaved from each wafer. Four were annealed for 2 hours in a tube furnace with a flowing Ar atmosphere $\left(2000 \mathrm{ml} \mathrm{min}^{-1}\right)$ at temperatures of $450^{\circ} \mathrm{C}, 500^{\circ} \mathrm{C}, 550^{\circ} \mathrm{C}$, and $600^{\circ} \mathrm{C}$, leaving one piece in the as-deposited state. The chosen temperature range straddles the typical bulk glass transition temperature of $560-580^{\circ} \mathrm{C}^{4,25,27}$ therefore significant variation in the films' structural and optical properties can be expected.

Figure 1 shows optical microscope images of the as-deposited and annealed GLS and GLSO thin films. The higher oxide content of GLSO yields a material with improved thermal stability, as indicated by the constant color of the annealed films. In contrast, there is a large blue shift in the color of the GLS films. ${ }^{\dagger}$ Under closer inspection (100x), the annealed films were found to be free of strain-induced cracking, despite the mismatched thermal expansion of GLS $\left(\sim 10 \pm 0.5 \times 10^{-6}{ }^{\circ} \mathrm{C}^{-1}\right)^{26}$ and silica $\left(\sim 0.55 \times 10^{-6}{ }^{\circ} \mathrm{C}^{-1}\right)$. Images of the films annealed at the highest temperatures $\left(550^{\circ}, 600^{\circ}\right)$ revealed the presence of strongly scattering point defects, which were assumed to be crystallized glass; an assumption supported by the fact that these defects were far more pronounced in GLS due to its lower oxide content.

\footnotetext{
${ }^{\dagger}$ The initial color difference between the as-deposited GLS and GLSO is primarily due to the different film thickness, rather than the small index change.
} 

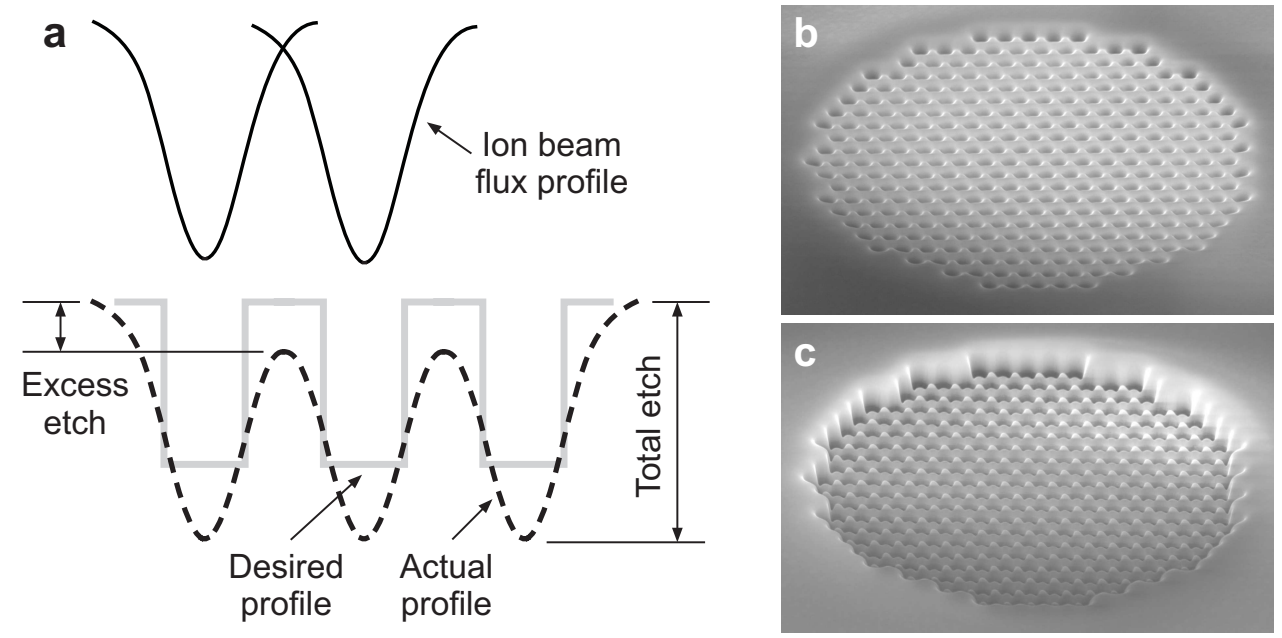

Figure 2. Excess etch in dense FIB-milled features. (a) Ion flux and the difference between actual and desired etch profiles. Adapted from Cryan et al. ${ }^{28}$ (b) and (c) A hexagonal lattice of holes milled in $\mathrm{Ta}_{2} \mathrm{O}_{5}$ with air fill factors of 0.23 and 0.58 , respectively.

Broadband variable-angle ellipsometry of the GLS films suggested that changes in both refractive index and film thickness were responsible for the variable film color. We suspect that the weak bonding of chalcogenides, volatility of sulfur compoounds, ${ }^{6}$ and tendency towards crystallization are the cause of these changes. Additional compositional analysis (EDS/SIMS) and stylus profiling is necessary to provide conclusive evidence of the suspected material losses and structural alterations.

\subsection{Optimizing the FIB Milling Process}

Due to the practical difficulties in patterning GLS-based glasses (see Sec. 2.1), we chose to fabricate our structures using a Zeiss NVision 40 focused ion beam (FIB) controlled by a Raith Elphy Quantum lithography system. However, while FIB milling is conceptually straightforward, there are several factors that complicate the milling of the closely-spaced nanoscopic holes characteristic of PhCs. Chief among these are the Gaussian flux profile of the focused beam and redeposition of sputtered material. The former leads to heavy 'excess etch' between the holes, as shown in Fig. 2a. ${ }^{28}$ For any given beam current (spot size), the excess etch becomes progressively worse as the proximity or depth of the holes increases. Figure $2 b$ and $c$ demonstrate the severity of excess etch for hexagonal PhCs milled in $\mathrm{Ta}_{2} \mathrm{O}_{5}$ with a $150 \mathrm{pA}(29 \mathrm{~nm})$ beam, a period of $a=400 \mathrm{~nm}$, and hole radii of $r=0.25 a$ and $r=0.4 a$. Although lower beam currents can significantly reduce excess etch, they lead to impractically long write times for large area structures.

Redeposition of sputtered material, combined with the Gaussian beam profile, make vertical sidewalls extremely difficult to achieve. Vertical sidewalls are critical in $\mathrm{PhC}$ slabs to preserve the transverse symmetry and minimize losses. To date, the most vertical sidewalls in FIB-milled PhCs have been fabricated using gas-assisted etching (GAE) with either $\mathrm{XeF}_{2}{ }^{29}$ or $\mathrm{I}_{2} \cdot{ }^{30}$ However, GAE, although available in our system, is not well-suited to etching more complex multi-component glasses such as GLSO. Alternative ways to improve the hole profile include adjusting the beam parameters and scan pattern, ${ }^{31}$ using hard masks, ${ }^{28}$ and milling through a suspended membrane ${ }^{31,32}$ to allow sputtered material to be ejected in both directions.

In Fig. 3 we demonstrate the effect of separately adjusting the beam current and the number of milling passes (loops) while keeping the total area dose constant at $0.12 \mathrm{C} \mathrm{cm}^{-2}$. Assuming only singly ionised $\mathrm{Ga}^{+}$ions, this is equivalent to approximately $1.92 \times 10^{18}$ ions $\mathrm{cm}^{-2}$. All holes are milled as concentric rings of pixels separated by $16 \mathrm{~nm}$ along the circumference of each ring. This scan path is similar to the spiral scanning used by Hopman et al. ${ }^{31}$ and radically improves hole circularity over simple raster scanning. The test patterns are hexagonal $\mathrm{PhCs}$ milled into $\mathrm{SiO}_{2}$ with $a=400 \mathrm{~nm}$ and $r=0.2 a$. Although these images are for $\mathrm{SiO}_{2}$, it was found that differences 

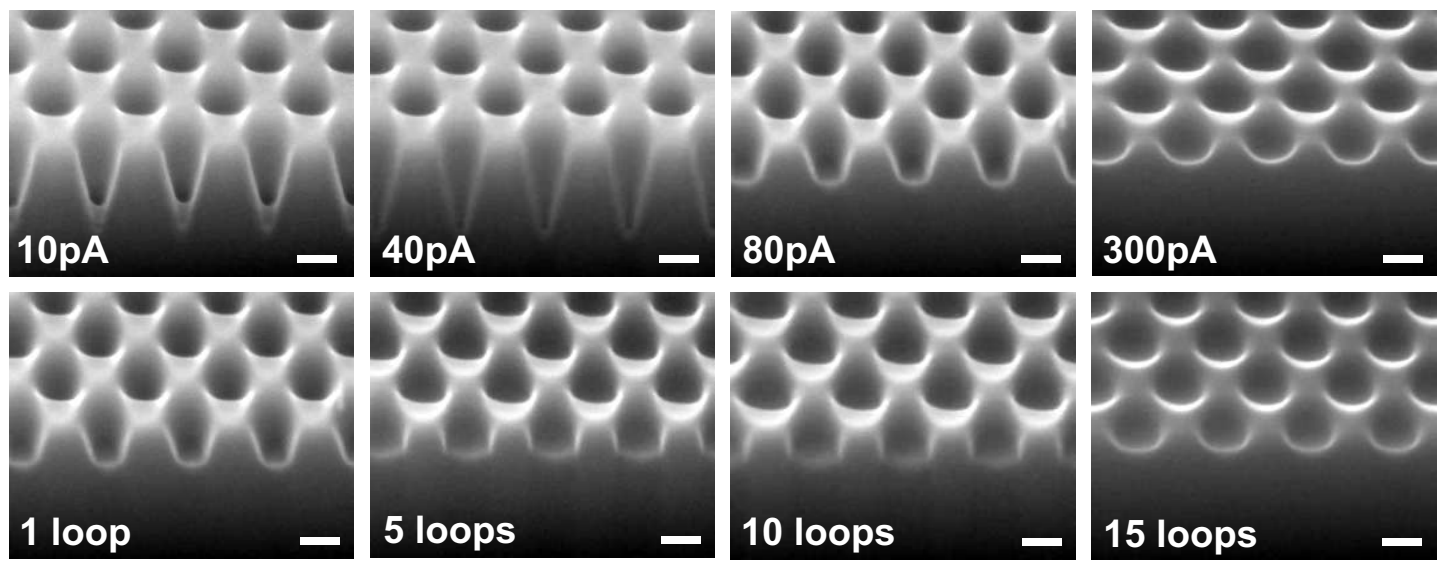

Figure 3. Hexagonal PhCs milled in $\mathrm{SiO}_{2}\left(a=400 \mathrm{~nm}, r / a=0.2,0.12 \mathrm{C} \mathrm{cm}^{-2}\right)$. Top row: effect of varying the beam current (spot size) for single pass milling. Bottom row: effect of varying the number of milling passes (loops) for a fixed beam current of $80 \mathrm{pA}$. Scale bar in all SEM images is $200 \mathrm{~nm}$.

were negligible for test patterns in GLSO, largely because of their amorphous nature and the subsequent lack of ion channeling effects.

The top row of Fig. 3 shows FIB cross sections of holes $(r=80 \mathrm{~nm})$ milled in a single pass for beam currents (spot sizes) of 10pA (13nm), 40pA (19nm), 80pA (22nm), and 300pA (38nm). The centre is the deepest point in all cases thus indicating that the Raith system scans from the outermost to the innermost ring of each hole. Varying the beam current for a fixed total dose using a single pass is equivalent to varying the pixel dwell time. Lower beam currents (long dwell times) result in deep holes with tapered sidewalls due to redeposition within the hole, milling at high incident angles, and containment of deflected ions. ${ }^{33}$ Higher currents (shorter dwell times) create a more catastrophic collision cascade, but maintain a level milled surface. In general, for a fixed dose, higher beam currents result in shallower holes. The $80 \mathrm{pA}$ beam clearly gives the closest approximation to the ideal square wave profile.

Long dwell times on a single pixel produce high-aspect ratio holes with limited mass transport. Multi-pass milling strategies are known to aid the removal of sputtered material and avoid re-deposition. However, for a given area dose, there is a limit to how many passes can be used before the pixel dwell time becomes too short for the beam blanker's scan electronics. ${ }^{31}$ Very high numbers of passes will also tend to exacerbate inter-hole milling. The bottom row of Fig. 3 shows FIB cross sections of holes milled with a fixed beam current of 80pA and a total dose of $0.12 \mathrm{C} \mathrm{cm}^{-2}$ spread over $1,5,10$, or 15 passes (loops). The milling efficiency decreases as the number of passes increases, as evidenced by the very shallow holes for a 15 pass strategy. However, a moderate number of passes leads to improved sidewall angles thanks to less redeposition. The sidewall angle for both 5 and 10 pass milling is greater than $80^{\circ}$. Five pass milling with an $80 \mathrm{pA}$ beam was chosen as the optimal milling strategy for our PhCs since the lower number of passes reduces both the cumulative beam settling time and inter-hole milling.

\subsection{Photonic Crystals in GLSO}

Fifteen hexagonal PhCs were milled into a piece of Er:GLSO coated thermally-oxidized silicon that was annealed at $500^{\circ}$. Each $\mathrm{PhC}$ was circular with a diameter of $100 a$. The period $(a)$ varied from $300 \mathrm{~nm}$ to $580 \mathrm{~nm}$ in $20 \mathrm{~nm}$ steps and $r / a$ was held constant at 0.2 . The patterns were milled at a working distance of $5.1 \mathrm{~mm}$ with an $80 \mathrm{pA}$ $(22 \mathrm{~nm})$ beam and a total dose of $0.12 \mathrm{C} \mathrm{cm}^{-2}$ delivered in 5 passes. This dose was sufficient to mill holes passing entirely through the doped glass. Figure 4 shows representative SEM images for patterns with periods of 300nm, $400 \mathrm{~nm}$, and $500 \mathrm{~nm}$. The structure was well-defined for $a>380 \mathrm{~nm}$, but suffered from significant excess etch for smaller lattice periods. 

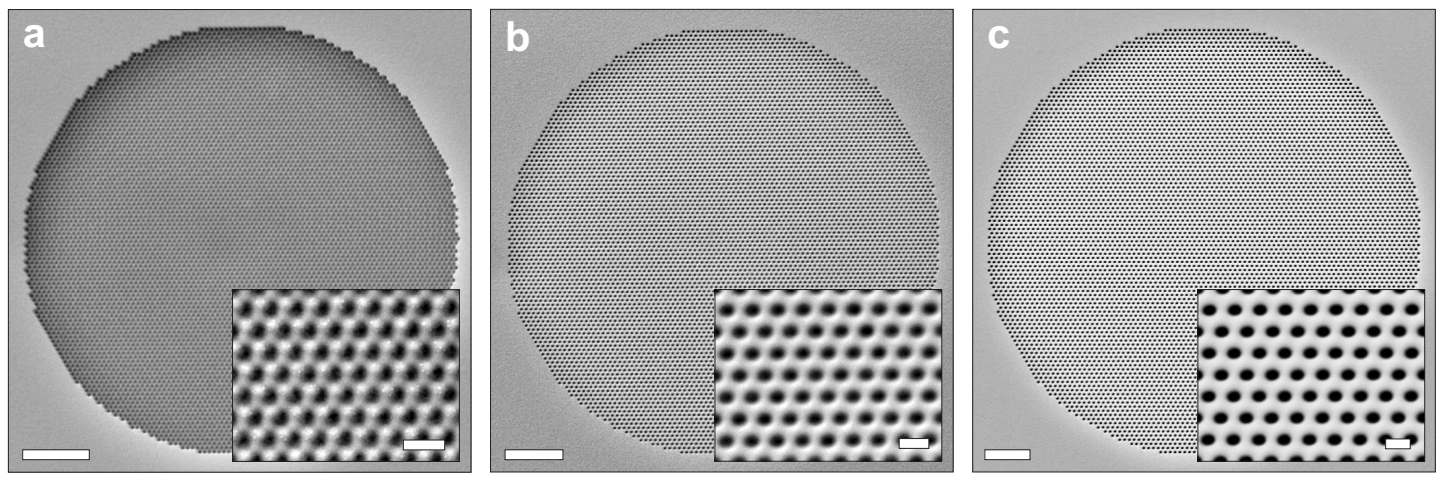

Figure 4. Hole-type PhCs milled into a 150nm thick film of Er:GLSO with a focused ion beam. (a,b,c) Hexagonal PhCs with periods of $a=300 \mathrm{~nm}, a=400 \mathrm{~nm}$, and $a=500 \mathrm{~nm}$, respectively. Scale bars in the large images (insets) are $5 \mu \mathrm{m}$ $(500 \mathrm{~nm})$.

\section{MEASUREMENT OF UPCONVERSION FLUORESCENCE}

\subsection{Experimental Setup for Fluorescence Measurements}

Upconversion fluorescence was measured with an optical microscope fiber-coupled to a spectrometer via a fluorescence excitation and emission filter cube. The light path inside the microscope is shown in Fig. 5. The two pump wavelengths of $808 \mathrm{~nm}(\mathrm{CW}, \max .200 \mathrm{~mW})$ and $980 \mathrm{~nm}(\mathrm{CW}$, max. $100 \mathrm{~mW})$ were provided by cooled and collimated laser diodes fixed to a kinematic mount for beam alignment. A $45^{\circ}$ dichroic mirror permitted transmission of both pump wavelengths (750-1100nm passband) whilst redirecting visible and infrared emission from the sample $(350-720 \mathrm{~nm}$, IR above $1100 \mathrm{~nm})$. The sample fluorescence was focused onto the tip of a multicore optical fibre coupled to the $250 \mu \mathrm{m}$ entrance slit of the spectrometer. Short-pass emission filters (OD4) were used to provide sufficient rejection of stray light at the excitation wavelengths and permit long integration times. Inside the spectrometer the visible fluorescence was dispersed by a 300 lines $\mathrm{mm}^{-1}$ grating blazed at $500 \mathrm{~nm}$, and detected by a silicon CCD array $(1024 \times 1024)$ TEC-cooled to $-70^{\circ} \mathrm{C}$.

Mounting the laser assembly on a separate double port attachment rather than the episcopic illuminator allowed easy switching between optical imaging and fluorescence measurements, and prohibited observation of the laser beam through the eyepiece. The laser power was controlled by ND filters and continually monitored by measuring the power reflected from the back of the dichroic mirror. Measurements at the sample were taken beyond the focal plane of the objective lens to avoid any nonlinear detector response due to the high intensity of the focused laser. A $50 \mathrm{x}$ microscope objective $(\mathrm{NA}=0.8)$ was found to offer the best compromise between throughput and spot size.

\subsection{Upconversion Fluorescence in Thermally Annealed Films}

Figure 6 presents the fluorescence spectrum of the unpatterned Er:GLSO films as a function of post-deposition annealing temperature for pump wavelengths of $808 \mathrm{~nm}$ and $980 \mathrm{~nm}(100 \mathrm{~mW} \mathrm{CW})$. The fluorescence signal was maximized by adjusting the focus between short 10s exposures. The final measurements presented below were obtained with much longer 90s integration times.

The shape of the fluorescence spectra and variation with annealing temperature are similar for both pump wavelengths, although a slightly different population of states is evident from the more prominent blue-shifted shoulder on the $550 \mathrm{~nm}$ peak for the $980 \mathrm{~nm}$ pump. There is no observable emission for the as-deposited films, even for much longer integration times. The visible fluorescence becomes measurable at the lowest annealing temperature and steadily increases up to a maximum at $550^{\circ} \mathrm{C}$, which roughly coincides with the glass transition temperature of the bulk material. ${ }^{34}$ Higher annealing temperatures result in partial crystallization of the films and quenching of the RE emission. The most notable difference between the two pump wavelengths is the $3.7 \mathrm{x}$ stronger signal for $808 \mathrm{~nm}$. The peak absorption cross section of $\mathrm{Er}^{3+}$ in non-oxide GLS at $805 \mathrm{~nm}$ is nearly half that at $984 \mathrm{~nm}\left(0.291 \mathrm{pm}^{2}\right.$ cf. $\left.0.537 \mathrm{pm}^{2}\right),{ }^{35}$ suggesting that upconversion from ESA at $\sim 800 \mathrm{~nm}$ is far more efficient than ESA and ET at 980nm. 


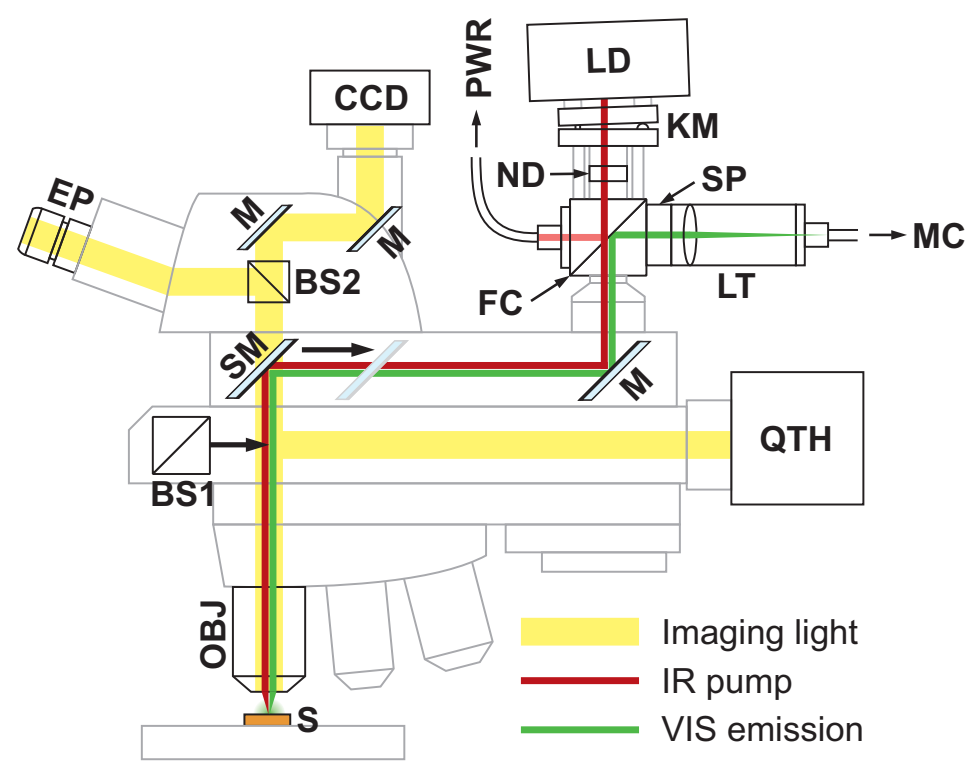

Figure 5. Light path inside the fluorescence microscope. Components are: laser diode head (LD), kinematic mount $(\mathrm{KM})$, filter cube with dichroic beamsplitter (FC), lens tube $(f=50 \mathrm{~mm})(\mathrm{LT})$, short pass filters (SP), ND filters (ND), fibre to optical power meter (PWR), fiber to monochromator/detector (MC), quartz tungsten halogen lamp (QTH), imaging camera (CCD), imaging eyepiece (EP), mirror (M), sliding mirror (SM), 50:50 movable beamsplitter (BS1), 80:20 beamsplitter (BS2), 50x objective (OBJ), and the sample under test (S).
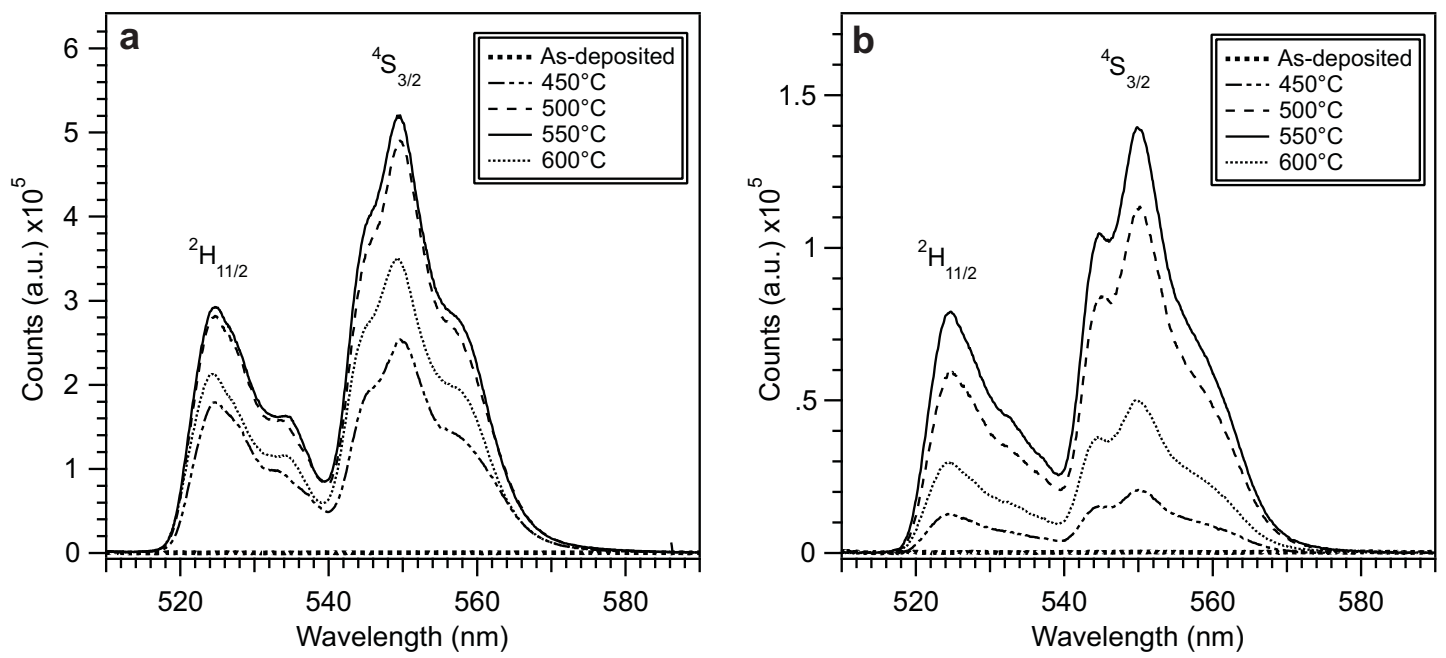

Figure 6. Upconversion fluorescence for a $150 \mathrm{~nm}$ thick film of $0.2 \mathrm{wt} \%$ Er:GLSO on a thermally-oxidized Si wafer with varying anneal temperatures pumped at (a) $808 \mathrm{~nm}$ and (b) $980 \mathrm{~nm}$. The optical power for each pump wavelength was adjusted to be $100 \mathrm{~mW}(\mathrm{CW})$ at the sample. 
Looking at the energy level diagram for $\mathrm{Er}^{3+}$ it is clear that pumping at $808 \mathrm{~nm}$ is resonant with ESA transitions from all three intermediate states: ${ }^{4} I_{9 / 2} \rightarrow{ }^{2} H_{9 / 2},{ }^{4} I_{11 / 2} \rightarrow\left\{{ }^{4} F_{3 / 2},{ }^{4} F_{5 / 2}\right\}$, and ${ }^{4} I_{13 / 2} \rightarrow\left\{{ }^{2} H_{11 / 2},{ }^{4} S_{3 / 2}\right\}$. The first two transitions result in population of states higher than the desired $\left\{{ }^{2} H_{11 / 2},{ }^{4} S_{3 / 2}\right\}$ states, thus requiring relaxation through further radiative or non-radiative transitions. However, the last transition indicates that a pump wavelength of $808 \mathrm{~nm}$ directly promotes ions from the favored ${ }^{4} I_{13 / 2}$ state to the levels responsible for green emission. Taken together, these transitions imply that efficient monochromatic upconversion pumping is possible at $808 \mathrm{~nm}$. The alternative solution to increase pump absorption is the common method of co-doping with sensitizing $\mathrm{Yb}^{3+}$ ions to significantly increase pump absorption on the ${ }^{4} I_{15 / 2} \rightarrow{ }^{4} I_{11 / 2}(980 \mathrm{~nm})$ transition. ${ }^{2}$

\section{CONCLUSIONS}

We have demonstrated the fabrication of hole-type photonic crystals in sputtered films of 0.2 wt\% Er:GLSO films using a focused ion beam. Optimization of the milled hole profile was achieved by varying the beam current and number of writing passes while using a concentric ring scan path. Upconversion from the unpatterned films was found to be most intense for an annealing temperature of $550^{\circ} \mathrm{C}$, which roughly coincides with the bulk glass transition temperature. Emission was also noted to be stronger at 808nm than 980nm due to ESA transitions from the $\left\{{ }^{4} I_{9 / 2},{ }^{4} I_{11 / 2},{ }^{4} I_{13 / 2}\right\}$ intermediate states. The modified density of states due to the presence of the photonic crystals is expected to result in enhanced upconversion by suppressing IR emission from the intermediate ${ }^{4} I_{13 / 2}$ manifold.

\section{ACKNOWLEDGMENTS}

The authors would like to acknowledge the financial support of the EPSRC, HEFCE, and the University of Southampton.

\section{REFERENCES}

[1] Schweizer, T., Hector, J. R., Brady, D. J., Wang, J., Hewak, D. W., Brocklesby, W. S., and Payne, D. N., "Visible upconversion in rare-earth doped gallium lanthanum sulphide based glasses," in [CIMTEC: 9th International Conference on Modern Materials \& Technologies], (1998).

[2] Oliveira, A. S., de Araujo, M. T., Gouveia-Neto, A. S., Neto, J. A. M., Sombra, A. S. B., and Messaddeq, Y., "Frequency upconversion in $\mathrm{er}^{3+} / \mathrm{yb}^{3+}$-codoped chalcogenide glass," Appl. Phys. Lett. 72, 753-755 (February 1998).

[3] Wang, J., Hector, J. R., Brady, D., Hewak, D., Brocklesby, B., Kluth, M., Moore, R., and Payne, D. N., "Halide-modified Ga-La sulfide glasses with improved fiber-drawing and optical properties for $\mathrm{Pr}^{3+}$-doped fiber amplifiers at 1.3pm," Appl. Phys. Lett. 71, 1753-1755 (September 1997).

[4] Frantz, J. A., Sanghera, J. S., Shaw, L. B., Villalobos, G., Aggarwal, I. D., and Hewak, D. W., "Sputtered films of $\mathrm{Er}^{3+}$-doped gallium lanthanum sulfide glass," Mat. Lett. 60, 1350-1353 (December 2006).

[5] Asal, R. and Rutt, H. N., "Optical properties of laser ablated gallium lanthanum sulphide chalcogenide glass thin films prepared at different deposition laser energy densities," Opt. Mater. 8, 259-268 (June 1997).

[6] Němec, P., Nazabal, V., Pavlišta, M., Moreac, A., Frumar, M., and Vlček, M., "Gallium-lanthanum-sulphide amorphous thin films prepared by pulsed laser deposition," Mater. Chem. Phys. 117, 23-25 (2009).

[7] Frantz, J. A., Shaw, L. B., Sanghera, J. S., and Aggarwal, I. D., "Waveguide amplifiers in sputtered films of $\mathrm{Er}^{3+}$-doped gallium lanthanum sulfide glass," Opt. Exp. 14, 1797-1803 (March 2006).

[8] Li, C. C., Kim, H. K., and Migliuolo, M., "Er-doped glass ridge-waveguide amplifiers fabricated with a collimated sputter deposition technique," IEEE Photon. Techn. Lett. 9, 1223-1225 (September 1997).

[9] Mairaj, A. K., Rizitos, C., Chardon, A. M., Smith, P. G. R., Shepherd, D. P., and Hewak, D. W., "Development of channel waveguide lasers in $\mathrm{Nd}^{3+}$-doped chalcogenide (Ga:La:S) glass through photoinduced material modification," Appl. Phys. Lett. 81, 3708-3710 (November 2002).

[10] Pan, W. J., Furniss, D., Rowe, H., Miller, C. A., Loni, A., Sewell, P., Benson, T. M., and Seddon, A. B., "Fine embossing of chalcogenide glasses: first time submicron definition of surface embossed features," $J$. Non-Cryst. Solids 353, 1302-1306 (2007). 
[11] Pan, W. J., Rowe, H., Zhang, D., Zhang, Y., Loni, A., Furniss, D., Sewell, P., Benson, T. M., and Seddon, A. B., "One-step hot embossing of optical rib waveguides in chalcogenide glasses," Microw. Opt. Techn. Let. 50, 1961-1963 (July 2008).

[12] Lian, Z. G., Pan, W., Furniss, D., Benson, T. M., Seddon, A. B., Kohoutek, T., Orava, J., and Wagner, T., "Embossing of chalcogenide glasses: monomode rib optical waveguides in evaporated thin films," Opt. Lett. 34, 1234-1236 (April 2009).

[13] DeCorby, R. G., Ponnampalam, N., Pai, M. M., Nguyen, H. T., Dwivedi, P. K., Clement, T. J., Haugen, C. J., McMullin, J. N., and Kasap, S. O., "High index contrast waveguides in chalcogenide glass and polymer," IEEE J. Sel. Top. Quant. 11, 539-546 (April 2005).

[14] Huang, C. C. and Hewak, D. W., "Silver-doped germanium sulphide glass channel waveguides fabricated by chemical vapour deposition and photo-dissolution process," Thin Solid Films 500, 247-251 (2006).

[15] da Silva, D. M., Kassab, L. R. P., Lẗhi, S. R., de Araújo, C. B., Gomes, A. S. L., and Bell, M. J. V., "Frequency upconversion in $\mathrm{Er}^{3+}$ doped $\mathrm{PbO}-\mathrm{GeO}_{2}$ glasses containing metallic nanoparticles," Appl. Phys. Lett. 90, 081913 (February 2007).

[16] Bykov, V. P., "Spontaneous emission in a periodic structure," Sov. Phys. JETP 35, 269-273 (1972).

[17] Noda, S., Fujita, M., and Asano, T., "Spontaneous-emission control by photonic crystals and nanocavities," Nat. Photon. 1, 449-458 (2007).

[18] Li, Z.-X., Li, L.-L., Zhou, H.-P., Yuan, Q., Chen, C., Sun, L.-D., and Yan, C.-H., "Colour modification action of an upconversion photonic crystal," Chem. Commun. , 6616-6618 (2009).

[19] Zhang, F., Deng, Y., Shi, Y., Zhang, R., and Zhao, D., "Photoluminescence modification in upconversion rare-earth fluoride nanocrystal array constructed photonic crystals," J. Mater. Chem. 20, 3895-3900 (2010).

[20] Markowicz, P., Friend, C., Shen, Y., Swiatkiewicz, J., Prasad, P. N., Toader, O., John, S., and Boyd, R. W., "Enhancement of two-photon emission in photonic crystals," Opt. Lett. 27, 351-353 (March 2002).

[21] St. J. Russell, P., "Photonic band gaps." Physics World (August 1992).

[22] Withnall, R., Ireland, T. G., Martinez-Rubio, M. I., Fern, G. R., and Silver, J., "Rare-earth element antistokes emission from three inverse photonic lattices," J. Mod. Opt. 49(5/6), 965-976 (2002).

[23] Bordas, F., Steel, M. J., Seassal, C., and Rahmani, A., "Confinement of band-edge modes in a photonic crystal slab," Opt. Exp. 15, 10890-10902 (August 2007).

[24] Lu, T., Yang, L., VanLoon, R. V. A., Polman, A., and Vahala, K. J., "On-chip green silica upconversion microlaser," Opt. Lett. 34, 482-484 (February 2009).

[25] Curry, R. J., Mairaj, A. K., Huang, C. C., Eason, R. W., Grivas, C., and Hewak, D. W., "Chalcogenide glass thin films and planar waveguides," J. Am. Ceram. Soc. 88(9), 2451-2455 (2005).

[26] Mairaj, A. K., Optical waveguides and lasers in improved gallium lanthanum sulphide glass, PhD thesis, Optoelectronics Research Centre (2003).

[27] Schweizer, T., Rare-earth-doped gallium lanthanum sulphide glasses for mid-infrared fibre lasers, PhD thesis, Optoelectronics Research Centre (1998).

[28] Cryan, M. J., Hill, M., Cortaberria-Sanz, D., Ivanov, P. S., Heard, P. J., Tian, L., Yu, S., and Rorison, J. M., "Focused ion beam-based fabrication of nanostructured photonic devices," IEEE J. Sel. Top. Quant. 11, 1266-1277 (November/December 2005).

[29] Cabrini, S., Carpentiero, A., Kumar, R., Businaro, L., Candeloro, P., Prasciolu, M., Gosparini, A., Andreani, C., De Vittorio, M., Stomeo, T., and Di Fabrizio, E., "Focused ion beam lithography for two dimensional array structures for photonic applications," Microelectron. Eng. 78-79, 11-15 (2005).

[30] Dale, G., Langford, R. M., Ewen, P. J. S., and Reeves, C. M., "Fabrication of photonic band gap structures in $\mathrm{As}_{40} \mathrm{~S}_{60}$ by focused ion beam milling," J. Non-Cryst. Solids 266-269, 913-918 (2000).

[31] Hopman, W. C. L., Ay, F., Hu, W., Gadgil, V. J., Kuipers, L., Pollnau, M., and DeRidder, R. M., "Focused ion beam scan routine, dwell time and dose optimizations for submicrometre period planar photonic crystal components and stamps in silicon," Nanotech. 18, 195305 (2007).

[32] Freeman, D., Madden, S., and Luther-Davies, B., "Fabrication of planar photonic crystals in a chalcogenide glass using a focused ion beam," Opt. Exp. 13, 3079-3086 (April 2005).

[33] Volkert, C. A. and Minor, A. M., "Focused ion beam microscopy and micromachining," MRS Bull. 32, 389-399 (May 2007). 
[34] Mairaj, A. K., Petrovich, M. N., West, Y. W., Fu, A., Harwood, D. W. J., Ng, L. N., Monro, T. M., Broderick, N. G., and Hewak, D. W., "Advances in gallium lanthanum sulphide glass for optical fibre and devices," in [SPIE Fiber Optic Sensor Technology II Part B], 4204, 278-285 (November 2000).

[35] Ye, C. C., Hewak, D. W., Hempstead, M., Samson, B. N., and Payne, D. N., "Spectral properties of $\mathrm{er}^{3+}$-doped gallium lanthanum sulphide glass," J. Non-Cryst. Solids 208, 56-63 (1996). 